\title{
What we know about acid rain
}

\author{
Kenneth Mellanby \\ Acid Rain: A Review of the Phenomenon in the EEC \& Europe. \\ Compiled by Environmental Resources Limited. \\ Graham \& Trotman, Sterling House, 66 Wilton Rd, London SWI/Unipub, \\ POB 433, Murray Hill Station, NY 10157: 1983. Pp. 159. £12.50, \$23.
}

IN 1982 Environmental Resources Limited (ERL), a private consultancy in London, received a contract from the Commission of the European Communities (EEC) to survey the problem of "acid rain". ERL rightly interpreted their brief to include all acid pollution produced by industry and the burning of fossil fuels. They also considered ozone, another pollutant of industrial origin, which may have its own harmful effects but which may also play a part in the generation of strong acids from other chemicals in the atmosphere.

The result, now published in book format, is a succinct and practical account, summarizing a vast amount of information in 160 pages, which include 15 of references. This bibliography is selective, but contains most useful publications up to 1982 . With this volume available, there is no longer any excuse for journalists to continue to produce the uninformed nonsense they have inflicted on the public in recent months.

It is clear that when we are considering the direct phytotoxic effects of acid emissions, it is when they are in the form of gases, and to a lesser extent, of particles, that they are important. It is dry deposition that does the damage. This is something with which we have been familiar for more than a hundred years, but recently opinions may have become confused by allegations that polluted rainwater - "acid rain" may be even more important. ERL give all the facts to allow the reader to disentangle the effects of dry and wet deposition. They make clear that, as far as direct damage to foliage and buildings is concerned, the acid rain commonly falling in the south of England has little harmful effect. Its acidity is thus something of a red herring, even though it is the property which has caused the greatest public disquiet.

Acute phytotoxicity in Britain has in fact greatly decreased in recent years, though this point is hardly made in the report. The improvement is generally attributed to the Clean Air Act of 1956, but was mainly caused by the substitution of clean, convenient natural gas for dirty coal in homes and industry. It has also been achieved by blowing the flue gases from coal-fired generating stations (still the biggest sources of sulphur dioxide) up tall chimneys so that they are mixed with air and thus diluted below the levels where plants are severely harmed.

This policy, obviously beneficial to Britain, has been severely criticized elsewhere. For instance a recent Swedish leaflet, handed out to tourists visiting that country, alleged that Britain is being selfish, reducing pollution at home by increasing it abroad. Fortunately this is not strictly true. Even with low chimneys only a small (though very damaging) amount of sulphur dioxide is deposited locally; most of it mixes into the atmosphere. So though higher chimneys produce important local reductions of pollution, they do not put much more sulphuric acid into the atmosphere; thus the gaseous concentrations a hundred or more miles from source are much the same whatever the height at which the emission originally took place.

\section{The Swedish countryside's secret: The acid rain is slowly killing it.}

Sulphur and nitrogen from the combustion of oil and coal fall with the rain, in the form of acid. This acid damages both the land, and the water. 18,000 lakes in Sweden have already been damaged by acid rain. Thousands of them are practically dead. If nothing is done, many more will soon be badly affected. The soil, the forests, and the subsoil water are also being dam. aged.

Acidification is our worst single environment problem. And yet many people are still unaware of this. It cannot be seen in the countryside. An acidified lake looks clean and clear, even if it has been poisoned to death.

Acidification is an international environment problem. That is why we say that what is happening in the Swedish countryside matters to you. Air pollutants are carried across countries, and across national frontiers, by the winds. Three quarters of the sulphur falltiers, by the winds. Three quarters of the sulphur
ing over Sweden originates from other countries.

The only way of saving our countryside from acidification is to reduce radically discharges in Europe, primarily of sulphur. This can only be achieved by inter. national collaboration. And that is why we are concerned to tell you about acidification - by making the problem known in your own country, you will be helping us to solve it!

Acid rain and international relations - detail from a leaflet, intended for tourists, issued by Swedish conservation and angling bodies.

Until recently, it was thought that the policy of "dilute and disperse" had been completely successful, both locally and on a global scale. However, we now find that some damage may occur to crops and trees from levels of gases below those where acute damage is evident. This seems particularly true when the oxides of sulphur and nitrogen mix with ozone. Nevertheless this is still a local problem, occurring within a radius of perhaps up to two hundred miles from the pollution source. Further off, with greater dilution, no further direct damage to plants or to buildings occurs. Until recently we thought that these very dilute gases were completely harmless, and that the pollution problem had largely been solved.

Unfortunately, it has not proved to be so simple. In Scandinavia, for instance, the levels of sulphur dioxide, a substantial part of which comes from Britain, are indeed low, and cause no direct damage to plant life. In fact very delicate foliose lichens, well known biological indicators of air pollution, flourish. But lakes and rivers are becoming acidic, fish are disappearing, and in some parts of Europe (though not in Scandinavia) trees appear to be damaged. This is all blamed on acid rain, and as the chemicals producing the acidity blow from one country to another, the problem is an international one.

A major cause of damage is believed to be that as the air passes from one area to another, perhaps taking several days to do so, the sulphur dioxide is transformed slowly to sulphate and sulphuric acid. This is washed out of the air, and may then acidify lakes, streams and soils where calcium levels are low and there is poor buffering. The rain itself, even when it contains sulphuric acid, is so dilute that it has no direct phytotoxicity; it is the indirect effect on the soil which is thought to be important.

But there are still many uncertainties. We simply do not understand the quantitative relationships between emissions and their environmental effects. One American report showed a linear relationship, with damage closely correlated with the amounts of pollution emitted. European studies have shown no such correlation. This disparity may be because different phenomena are being investigated. The local effects within the UK of air pollution generated in Britain are roughly proportional to emissions, certainly within a radius of a couple of hundred miles from source. But more distant effects, involving chemical transformations whose rate depends on several factors, cannot be determined so easily.

The value of this publication is that it summarizes the existing information, and shows how little we know and where research is needed. I cannot describe the situation better than by quoting from the summary:

However, it has not been unequivocably established that these environmental impacts are caused by acid pollutant emissions, nor is the relative importance of other factors properly identified. Also considerable further investigations are required to understand the mechanism involved. Nevertheless circumstantial evidence would suggest that acid emissions and their subsequent chemical transformation and precipitation are at least a partial contributory cause to the observed effects and may be giving rise to as yet unidentified impacts, some of which could be irreversible.

Kenneth Mellanby is Chairman of the Working Group on Acid Rain of the Watt Committee on Energy. This committee represents 60 British professional engineering and scientific organizations. 